Health \& Medicine | Akos Koller

\section{A blow to the head impairs the autoregulation of brain blood flow}

Neural consequences

Billions of cells make up the
human brain. To keep this super-
computer functioning, a carefully
autoregulated blood supply
provides oxygen and nutrients
and removes waste products
from the brain cells. In addition,
cerebral circulation is involved
in the regulation of the pressure
inside the cranium. But what
happens if this autoregulatory
process is disrupted? Building
on years of research into the
microcirculation, Professor
emeritus Akos Koller at
Semmelweis University,
Hungarian University of Sports
Science (both in Budapest,
Hungary), and New York Medical
College (Valhalla, USA) explores
the impact that traumatic
brain injury can have on blood
circulation in the brain and the
mechanisms underlying this
dysregulation.

The human brain acts as a super- their supportive vasculature system; computer to monitor and control the two are intrinsically linked. It's like a every single process within the human body. To help it achieve this, the cells, all of which are connd 00 bilion cells with trillions of symapses (junctions that allow transmission of chemical or electrical signals between two cells). To survive, each of these cells requires a precise supply of oxygen and energy as well as removal of waste products. and carbon dioxide. These special tasks are carried out by millions of blood vessels within the brain: the cerebral blood vasculature.

Professor emeritus Akos Koller at Semmelweis University, the Hungarian University of Physical Education and Sport Sciences, Budapest, and New Yor Medical College explans that the vascular system of the brain is often often focuses on the neurons - brain cells - themselves. However, it is not possible for these cells to exist withot

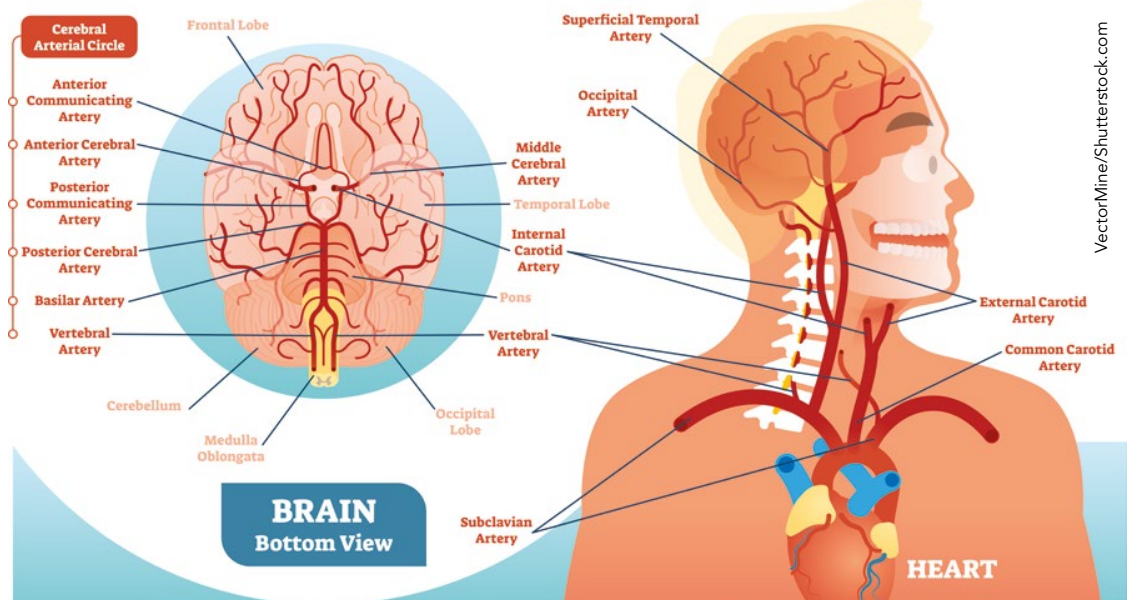
marriage. if one is not happy the oth refers to manovisc and which enable sufficient oxygen active and energy-demanding neurons and astrocytes - cell types found in the central nervous system.

It is important to remember that the amount of blood within the cerebral vasculature system is limited by the available space within the skull, the volume of which is - understandably unable to increase even if the perfusion pressure (i.e., blood pressure) increases. This constraint had been realised more than 200 years ago by Monro and Kellie, but the responsible mechanisms remain obscure. To avoid ncreases in intracranial pressure and thus impairment of bran be carefully autoralogutan primarily achieved by mechanosensitive vascular mechanism

\section{WO FORCES: PRESSURE} AND SHEAR STRESS One way vasomotor mechanisms through changes in the diameter of blood vessels. First the 'myogenic response' was recognised, which is activated by changes in blood pressure. If blood pressure increases, the smooth muscle in the outside layer of the blood vessels constrict, thereby creating resistance which ensures that increase

By carefully studying the literature, Koller's team however deduced that the myogenic response alone is not enough to explain the constant blood another mechanism that is triggered by changes in blood flow-related force in landmark study in 2011, Koller and his team discovered that increases of flow constrict isolated cerebral arteries, supporting their idea that flow sensitivity of cerebral vessels contributes to the autoregulation of cerebral blood flow. These mechanosensitive vasomotor mechanisms in the walls of the cerebral blood vessels 'measure' blood pressure (the force exerted by the blood on the vessel walls) and blood-flow-induced shear (due to the high speed of blood). They help to keep the pressure and flow relatively constant in the skull and thus blood circulation elsewhere in the body.

\section{TRAUMATIC BRAIN INJURIES}

If these mechanisms are damaged, for example due to a traumatic brain injury his can result in impaired autoregulation of blood flow. In turn, this can lead to increased pressure within tiny blood vessels (capillaries), fluid build-up as fuid out, and thus causes oedema and increased pressure within the skull. As the skull is unable to expand, this can lead to compression of the brain and impaired blood flow to brain tissues. To alleviate this pressure and accompanying headache, in some cases surgeons must open the skull, a method that een used by Inca Indians.

In the USA alone, traumatic brain injuries affect around 1.5 million people each These injuries caround $50,000 \mathrm{de}$

variety of events, such as accidents, falls, sport activities, and even shaken baby syndrome. Traumatic brain injuries can have severe consequences which are often due to the death of cells within the brain. Koller hypothesises that impaired autoregulation of cerebral blood supply may aggravate existing damage to

\section{HEAD TRAUMA LEADS}

TO IMPAIRED BLOOD CYRCULATON AND

DYSUNCTION OF THE BRAIN pressund colleagues showed that the

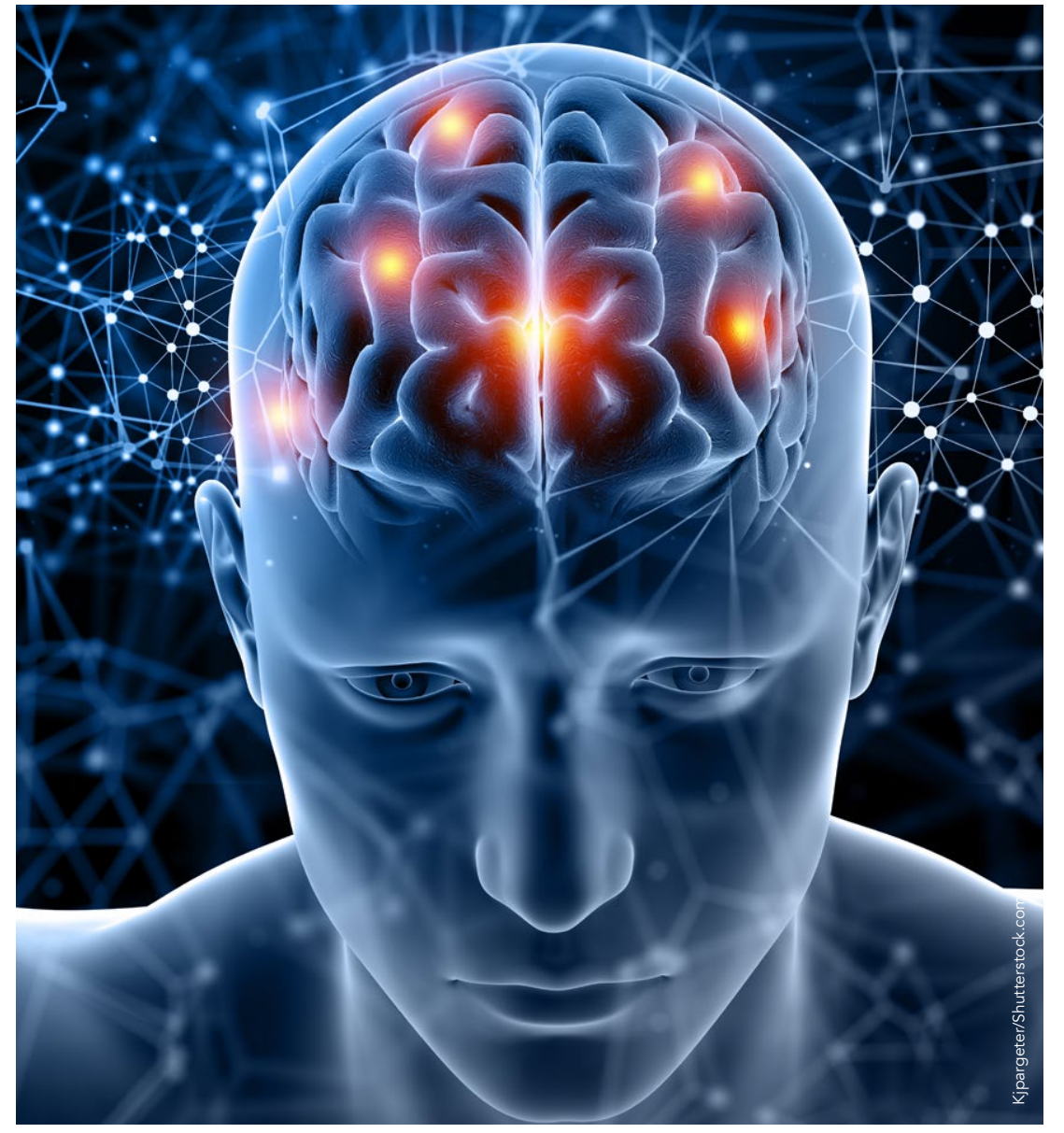

Blood flow to the brain must be carefully regulated - by changing the diameter of cerebral vessels -
because of the limited space available in the skull. Traumatic brain injury can impair this regulation and could result in neural dysfunction, such as loss of memories, cognitive deficiencies, and can even lead

To avoid increases in intracranial pressure and impairment of brain function, the cerebral blood flow must be carefully autoregulated - which is achieved by mechanosensitive vascular mechanisms.

become impaired after traumatic brain injury. Then, in recent studies, they used a rodent model and isolated arteries from the areas of the brain which had Their intricate pressure-flow chamber experiments enabled the team to carefully measure the characteristics of very small, individual cerebral blood vessels. In this way, they showed that increases in blood flow inside the vessels, which would normally result in constrictions, became substantially reduced after head trauma. Using pharmacological tools and prove the production of metabolites from a compound called arachidonic acid by a group of enzymes known as such as 20-HETE, are already known to play important roles in the constriction of blood vessels, through receptors tha cecognise hormone-like compounds called prostanoids as well as 20-HETE. Koller and colleagues showed that after is less a be to inery, cylochrome P450 acid meanin tabolise arachidonic produced Merat less 20 HETE is produced. Moreover, prostanoid 20-HETE. This meant that vessel 


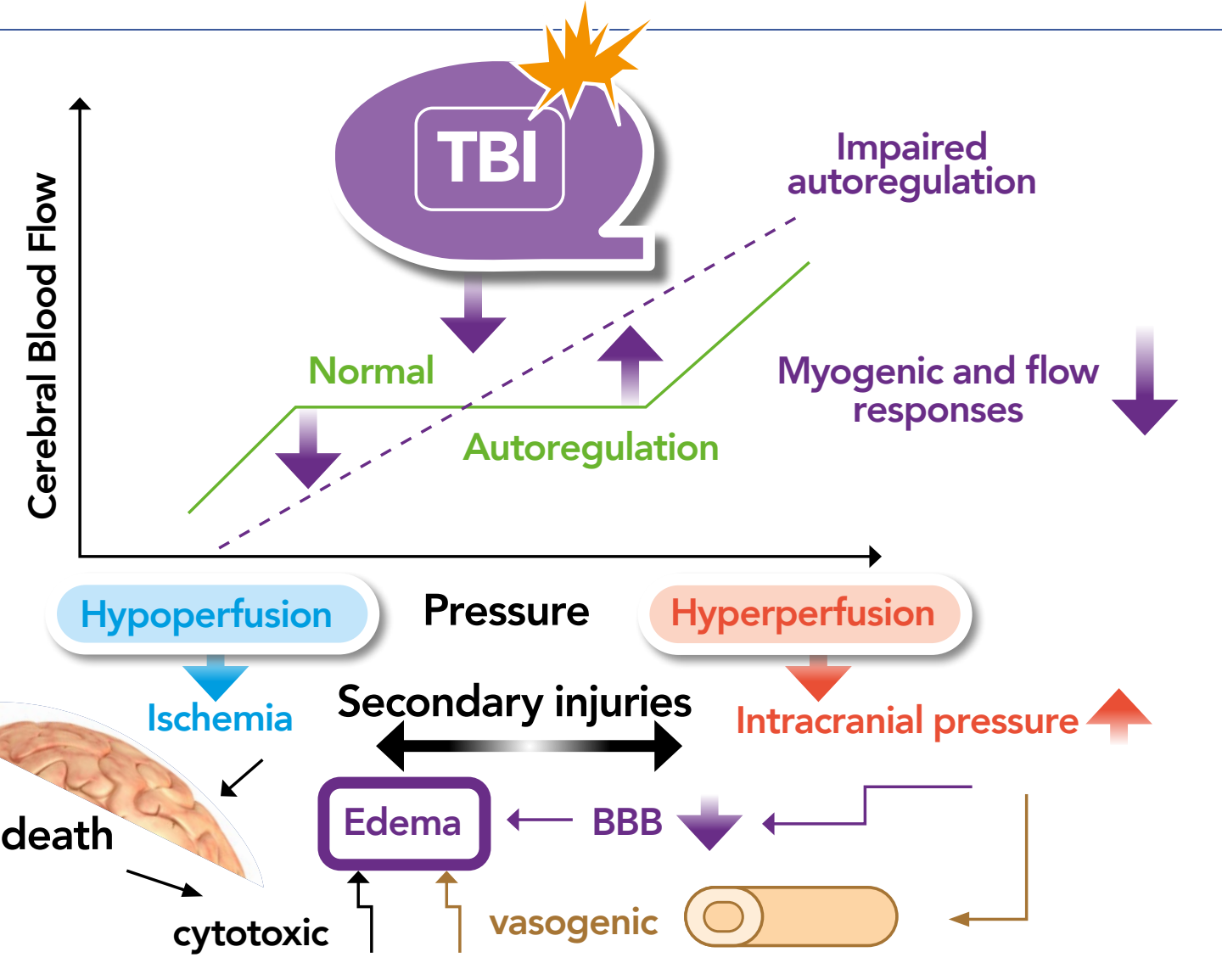

Autoregulation of cerebral blood flow is indicated by the flat portion of the green line. By impariting pressure and fllow sensitivity of vessels, traumatic brain

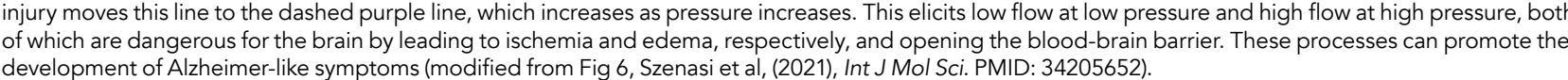

constrictions caused by changes in blood flow are impaired.

Their study also showed that after head injury, hydrogen peroxide produced by the "power houses' of the cell, the mitochondria, can interfere with ion channels in the vascular cell channels play an important role

in ensuring that vascular smooth muscle constricts muscle constricts
to increase blood pressure with the vessels.

Therefore, the impairment of these on channels may also contribute to impaired autoregulatory responses of cerebral vessels.

In addition, Koller explains that the impaired autoregulatory mechanisms of con vessels can play an important role in complications or single or reperive mid encephalopathy. This may also be due to

\section{the disruption of the blood-brain barrier (which normally prevents the movements of 'unwanted' drugs or toxic molecules} rest of the body), and failure to eliminate waste products from the brain, resulting waste products from the brain, resulting

together with physical therapy,

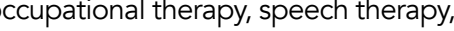
psychology/psychiatry, and social support

Nevertheless, identifying and understanding mechanisms associated Koller explains that the impaired targeted with drugs gulatory mechanisms of brain complications of single or repetitive therefore avoiding of complications injury. While there with the development of Alzheimer-like have been several animal studies diseases, and vascular dementia. no investigations have explored such possibilities in humans. Future studies Currently, there are no treatments __ led by Koller have new aims: to assess avaliable to restore autoregulatory autoregulatory responses of cerebrat vascular function after traumatic brain blood vessels in humans, in various injuries. Instead, therapies focus on disease conditions considering age, sex normalising factors known to impact and pregnancy, and then try restoring

\section{Behind the Research}

\section{Professor Akos Koller}

\section{Research Objectives}

Professor Koller investigas the microciculation inhealh and disease. His recent work explores traumatic-brain-

\section{Detail}

Professor Akos Koller

Medicine \& Department of Morphology-Physiology, Semmelweis University, 1085 Budapest, Hungary,

. .

Professor Koller graduated from Semmelweis Medical School in 1975, and continues to be on the Faculty until today Since 1982, he has been conducting research in microcirculation with Paul C Johnson at the University of Arizona, then from 1987 with Gabor Kaley at New York Medical College, Award of the European Society for Microcirculation.

Funding

-American Heart Association (AHA)

- National Institutes of Health (NIH)

- National Institute on Aging (NIA)

- The Marie Sklodowska Cure Actions SMARTER 7th

-

- National Research, Deal of End Innovation Office

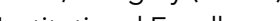

ersity of Sports Science

\section{Collaborators}

Peter Toth and Andras Buki (University of Pécs, Hungary) and Zoltan Ungvari Semmelweis University, Hungary and University of Oklahoma, OK, USA)

DD students: Nikolett Szarka, Annamaria Szenasi, and

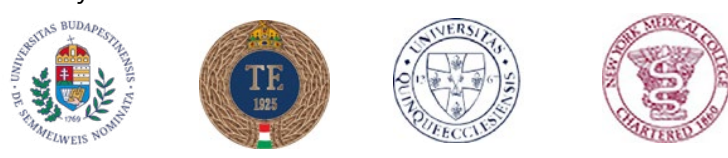

\section{References}

- Centers for Disease Control and Prevention (2022) Traumatic Brain Injury \& Concussion [online]. www.cdc.gov - Szenasi, A Amrein, K Czeiter, E, et al, (2021) Molecular Pathomechanisms of Impaired Flow-Induced Constriction of Cerebral Arteries Following Traumatic Brain Injury: A Potential Impact on Cerebral Autoregulation. Internation Journal of Molecular Sciences, 22(12), 6624. doi. org/10.3390/ijms22126624

- Szarka, N, Toth, L, Czigler, A, et al, (2019) Single Mild Traumatic Brain Injury Induces Persistent Disruption of the Blood-Brain Barrier, Neuroinflammation and Cognitive Decline in Hypertensive Rats. International Journal of Molecular Sciences, 20(13), 3223. doi.org/10.3390/ ijms20133223 - Toth, P, Szarka, N, Farkas, E, et al, (2016) Traumatic brain injury-induced autoregulatory dysfunction and spreading depression-related neurovascular uncoup Pathomechanisms, perspectives, and therapeutic and Circulatory Physiology, 311(5), H1118-H1131. org/10.1152/ajpheart.00267.2016

- Toth, P, Rozsa, B, Springo, Z, et al, (2011) Isolated huma and rat cerebral arteries constrict to increases in flow: role of 20-HETE and TP receptors. Journal of Cerebral Blood Flow and Metabolism, 31(10), 2096-2105. doi.org/10.1038 jcbfm.2011.74

\section{Personal Response}

What are the next steps required to learn more about how we could restore autoregulatory constriction

II At the moment I do not see how would it be possible to specifically send molecules to the brain to restore the vascular function of CYP450 and prostanoid receptors. What is possible, however, is to avoid anything that would "weaken' the autoregulatory function of cerebral vessels (some commonly used over the counter drugs). In addition exercise, known to increase blood pressure and flow, can also strengthen and 'train' this function. But first, I am planning to investigate whether or not cerebrovascular reactivity is impaired in preeclampsia (high blood press pregnat mothers. 